\title{
Brièveté, finitude et écritures
}

\author{
Nelson Charest \\ Université d'Ottawa
}

Dans son plus récent ouvrage, Gérard Dessons (2015, p. 31) souligne la faveur qu'ont reçue les formes brèves dans les études littéraires ces dernières années ${ }^{1}$. Il rappelle que notre époque multiplie les écritures courtes et rapides, que nous sommes submergés par des discours impromptus et souvent improvisés, que la vitesse est notre lot et que, en toute chose, le temps nous manque. Afin de s'ancrer dans cette réalité plus tangible, Dessons choisit de s'intéresser au «bref » plutôt qu'à la «brièveté »: «Plus précisément, je distinguerai la brièveté, terme abstrait désignant une question, un champ notionnel, et le bref, terme concret, indissociable de la réalité empirique des œuvres. » (2015, p.11) Or mon point de vue sera inverse, et je montrerai tout à l'heure comment Dessons, se limitant au «bref » dans le discours, se coupe

${ }^{1}$ Je détaille ici les ouvrages qu'il mentionne à la page susmentionnée : Formes brèves. De la gnômè à la pointe : métamorphose de la sententia. La Licorne, 1979; Messina, 1996; Foyard, 2001; Montandon, 1992; Leplâtre, 2005; Roukhomovsky, 2005. 
ainsi d'une part de «concret » et de «réalité » que permet de saisir la brièveté. Du reste, même si Dessons nominalise «le» bref, il ne peut faire autrement que de recourir constamment à son pendant, « la brièveté », et parfois dans un même développement².

Par ailleurs, Dessons ne se limite pas à la poésie mais il en vient, en fin de course, à énoncer cette remarque que j'endosserai pleinement et qui esquisse une relation particulière entre la poésie et la brièveté - sans reprendre, notons-le en passant, le lieu commun du poème comme « genre court» :

Sur un plan général, on peut donc définir le bref par ce moment où le langage passe en mode poème, où il quitte le régime du signe pour se mettre en régime rythmique. Étranger aux typologies des genres, le bref a pour enjeu l'individuation d'un sujet qui s'invente en inventant son langage [...]. En ce sens, chaque poème est bref à sa façon, chaque poème est poème d'inventer son bref, qui le définit comme subjectivation et valeur, comme manière. (2015, p. 143)

Si donc le bref est une modalité discursive parmi d'autres, on doit penser, à l'inverse, que la modalité «poétique » a besoin, elle, de la modalité «bref » pour trouver son caractère propre. Et que cela peut se faire, comme le dit Dessons, hors des voies d'une étude des genres.

\section{Du bref à la brièveté}

Et tout comme Dessons, je pourrais commencer cette analyse en précisant quelques éléments que j'ai exclus, des aspects ou types de textes que je juge non pertinents pour mon propos et qui nous entraîneraient ailleurs. Comme lui, je ne crois pas que le bref peut mener à la disparition du langage, comme dans ce « langage des anges » (Dessons, 2015, p. 13-16) qui se passerait de mots pour communiquer : la brièveté, peu importe l'angle sous lequel on la considère, est d'abord et avant tout une affirmation d'existence et on ne pourra jamais

\footnotetext{
2 «Si donc la brièveté est une problématique interne au domaine du langage, elle n'est pas pensable en dehors des questions théoriques dont l'enjeu est la discursivité. Dans cette perspective, tout traitement du bref apparaît comme nécessairement surdéterminé par des présupposés théoriques portant sur le langage et sur la littérature. » (Dessons, 2015, p. 27), je souligne.
} 
projeter le « peu» (ou le « concis», ou le « rapide», etc.) sur le « rien ». Comme lui encore, je m'élève contre l'association à mon sens beaucoup trop rapide entre la brièveté et le fragmentaire (Dessons, 2015, p.16-22), qui prend une ponctuelle ressemblance morphologique pour une identité, à la faveur, je crois bien, d'une mode qui les valorise tous deux au même moment. Un éclat de verre ne sera jamais dit « bref », et le chemin entre Ottawa et Montréal ne sera jamais qualifié de «fragmenté». Quant à la croyance si souvent entretenue que le haïku représente l'hypostasie de la brièveté (Dessons, 2015, p. 22-24), il faut la combattre sans en nier le présupposé, comme le fait je crois Dessons lui-même. Si tout poème participe de la brièveté, il n'y a pas de raison de la refuser au haïku (à condition, bien sûr, qu'il soit «poétique », ce qui n’est évidemment pas certain). Mais on devrait cesser de croire qu'elle s'y trouve là plus ou mieux qu'ailleurs.

Il reste à dire, toujours après Dessons, ce que peut avoir de contre-productif l'appellation «formes brèves » (2015, p. 25-32), qui entraîne vers un formalisme excessif, qui lui-même s'accompagne d'une attention par trop exclusive aux questions de dimension. Le «bref», ici plus qu'ailleurs, se limite le plus souvent au «court», et produit donc ce que Dessons appelle à juste titre un « bric-à-brac », tout et rien pouvant être jugé court, dans tous les genres comme dans toutes les formes de discours. Le problème est qu'après avoir reconnu ce problème, Dessons reste à mon sens trop timide, en se limitant à étudier des formes brèves moins étudiées (le proverbe, les «brèves », l'épigramme, ou la litote parmi les figures) plutôt qu'à adopter un autre point de vue sur la question. Je vais ici montrer comment cela devient possible, à partir précisément des deux principes qui guident l'argumentation de Dessons.

L'un des chapitres les plus importants de l'ouvrage de Dessons s'intitule « Le bref n'est pas le court», ce qui pose de façon on ne peut plus claire une distinction qui devenait nécessaire. Ce qui distingue la brièveté, selon Dessons, est qu'elle « apparaît historiquement 
liée à des problématiques de l'énonciation» (2015, p. 27). Soulignant que le français ne dispose que d'un substantif, la « brièveté », pour qualifier à la fois le « court» et le «bref», il s'engage à défaire la synonymie qui selon lui s'est installée entre ces deux termes: « contrairement à court, bref a une affinité forte pour le champ du langage » (Dessons, 2015, p. 46). La prolifération ces dernières années de l'expression «forme brève » serait selon lui le signe d'un nivellement progressif des deux notions et d'une perte de la spécificité discursive de la brièveté :

La dimension n'a pas toujours été un paramètre de la brièveté, que les théoriciens de la rhétorique cantonnaient dans les limites d'une économie discursive. En passant de l'ancienne brevitas à la moderne forme brève, la réflexion sur la brièveté est allée dans le sens d'un abandon de la discursivité. (Dessons, 2015, p. 48)

Plus loin il précisera :

Appliqués au langage, bref et court se distribuent en fonction de l'engagement ou du désengagement du locuteur par rapport au discours. Bref implique celui qui parle, lui assigne un lieu interne à sa parole, contrairement à court, qui implique un point de regard extérieur au discours en tant que réalisation subjective. (Dessons, 2015, p. 49)

Or on peut opposer deux objections à ces remarques. La première est que cette question de l'évolution lexicale, qui reprend pour l'essentiel un article de 1991, me semble hautement biaisée. Limiter la question de la dimension au seul syntagme «court», ou limiter la dimension du «bref» à la seule mesure temporelle, alors que le «court» désignerait une mesure spatiale, est toujours trop restrictif et ne recoupe pas des usages que les dictionnaires même anciens relevaient. Il serait plus juste de dire que la brièveté est marquée dès son origine par les deux sèmes de la dimension et du discours. Cette double valence, selon moi, doit être opposée au « court». Elle signifie qu'il existe deux régimes de la dimension, et qu'un de ces régimes se situe plus proprement dans le langage, que les objets de langage et les textes tout particulièrement le représentent et le révèlent mieux que toute autre chose. Comme Dessons, je crois que lorsque nous tentons de «mesurer » l'objet langagier avec des moyens 
qui lui sont étrangers, par toutes ces mathématiques qui sont assez courantes lorsqu'on y songe (nombre de caractères, de lignes, de pages, de syllabes, etc.), nous plaquons sur le bref une logique qui est propre au court. Mais on doit aller plus loin, et ne pas seulement considérer la brièveté sous l'angle du discours qui montre, par l'énonciation, l'interaction d'un sujet et d'un langage. Cela est vrai mais encore incomplet. Et cette incomplétude tient notamment au choix du «bref » plutôt que de la «brièveté », qui n'est pas seulement un choix de l'empirie, comme le croit Dessons; c'est aussi un parti-pris, posé avant même la recherche et les questions, qui amène à valoriser les manifestations écrites de la brièveté, dans l'optique d'une connivence avec le concis.

Car la brièveté déborde le simple départage entre dimension et discours. Par deux fois, mais comme avec un repentir, Dessons écarte de son étude des éléments qui lui auraient ouvert d'autres voies. Dans un premier temps, lorsqu'il évoque la possibilité d'une appréciation subjective de la brièveté. La brièveté serait ainsi liée à une perception plutôt qu'à une mesure objective, comme le court, ce qui élargirait le rôle du sujet dans son appréhension, dans la droite ligne de ce que défend Dessons. Mais il écarte cette voie, qui lui semble indémontrable :

Pour sortir de ce cercle vicieux d'une objectivation impossible du bref, il a été proposé de reverser la question du côté de la subjectivité perceptive : serait bref ce qui est perçu comme bref. Le problème, c'est qu'on ne règle pas un fantasme de l'objectivité en installant à sa place un fantasme symétrique, celui d'un subjectivisme de la réception. (Dessons, 2015, p. 30-31)

Certes, il ne s'agit pas de tomber dans des déclarations vagues du type « les goûts sont dans la nature » ni de laisser toute appréhension de la brièveté au jugement subjectif de chacun. Mais cela ne signifie pas qu'on doit pour autant écarter tout critère de perception, ce que Dessons lui-même semble faire quelques pages plus loin, dans ce que j'ai qualifié plus tôt de « repentir ». Il y est question alors du Protagoras de Platon et d'un dialogue entre ce dernier et 
Socrate, autour justement d'une «juste mesure » à observer dans les discours. Socrate reproche à Protagoras de s'approprier indûment l'échange en pratiquant systématiquement l'amplification dans ses réponses, alors que Socrate préfère les réponses courtes, qui permettent un échange plus juste et stimulant. Ce à quoi Protagoras répond ceci, non sans malice: «- Est-ce, maintenant, de la longueur jugée par moi nécessaire, est-ce de cette longueur que devront être mes réponses? ou bien d'une longueur dont tu seras juge? » (334 e, cité par Dessons, 2015, p.117) Non seulement Protagoras pose ainsi deux critères de longueur, selon la perception de deux sujets différents, mais aussi la question de l'autorité qui devra en juger; et c'est là, on le sent bien, à un autre défaut de l'échange que Protagoras fait référence, demandant implicitement à Socrate selon quelle autorité il se fait le seul juge de la juste mesure. Socrate n'ira pas dans cette voie et c'est pourquoi Dessons note ceci, qui entre en conflit, à mon sens, avec ce qu'il disait plus tôt :

Protagoras déplace le problème en rattachant la nécessité à la relation du sujet et du discours (le bref n'est pas bref relativement à une donnée objective ou idéale, à "quelque chose », mais par rapport à un sujet, à quelqu'un). C'est dans l'espace théorique laissé en suspens par la non-réponse de Socrate, que peut prendre place une réflexion sur le bref comme pensée critique de la subjectivation dans le langage. (2015, p. 119)

Je ne suis pas certain que ces deux citations de Dessons sont contradictoires, mais il reste qu'elles sont au mieux complémentaires, et que la deuxième apporte une nuance fondamentale. Certes, la brièveté n'est pas purement relative au jugement de chacun; mais de l'autre côté, toute volonté d'établir une brièveté objective et mesurable achoppera, parce que la brièveté se pense toujours à partir d'un foyer subjectif, et donc d'une perception.

\section{La mesure vive de la brièveté}

Pour nous en convaincre, je vais moi-même déplacer la question que pose ainsi Dessons, vers un autre texte de Platon. Car si Socrate ne répond pas à l'objection de Protagoras, du moins 
pas dans les termes que celui-ci pose, la réflexion de Platon y reviendra quant à elle, dans un de ses derniers textes, Philèbe, qui traite du plaisir. Pour établir ce qui peut procurer du bien et du bonheur dans la vie, Platon distingue quatre éléments ${ }^{3}$ : l'apeiron est l'illimité, l'indéterminé, tout ce qu'on introduit par des comparatifs comme «plus chaud», «trop froid», ou des termes positifs qui impliquent un comparatif, comme «doucement» ou « aigu »; ensuite le peras, qu'on associe à la limite, à la détermination, à tout ce qui introduit « le nombre »; troisièmement l'ousia, qu'on qualifie de «détermination de l'indéterminé », ce pourquoi Platon précise qu'il s'agit d'une «ousia advenue», pour insister sur un processus en cours et qui trouve son équilibre, dans la santé, la beauté, la vigueur, par exemple; et enfin on ajoute une quatrième catégorie qui est la cause qui génère la progression entre les trois précédents, qu'il nomme également le producteur ou le démiurge, et qu'il associe à la raison.

Je pense qu'on peut dès lors, à la lumière de ces distinctions, reprendre ce qui a été dit plus tôt. Lorsque Dessons condamne un fantasme du subjectivisme, il s'oppose à une conception qui ferait de la brièveté un simple apeiron, un simple indéterminé soumis à l'aléatoire. Il faut préciser ici que si Platon qualifie cette catégorie « d'illimité », c'est qu'elle entraîne à des comparaisons infinies : dire d'une chose qu'elle est « plus froide » pose toujours implicitement qu'une chose est «plus froide » que toutes celles qui sont « moins froides » jusqu'à ce que cette chose même devienne «moins froide » qu'une autre chose, qui elle sera «plus froide», et cela à l'infini. C'est bien ce que pose la difficulté de la brièveté comme indéterminé : dès qu'on établit qu'une chose est brève, on reste sous la menace que quelque chose de plus bref encore apparaisse, et l'ancien bref devient alors le long.

Or lorsque dans un deuxième temps Dessons endosse l'objection de Protagoras, c'est plutôt à l'ousia alors qu'il se réfère, à ce qu'on traduit précisément par la «juste mesure ».

\footnotetext{
${ }^{3}$ Les explications et les exemples se fondent ici sur Delcomminette, 2005.
} 
Mais nous ne sommes plus ici dans le cadre strict du discours, quoique le sujet soit toujours aussi présent dans l'équation, sinon d'autant plus. Car si l'ousia est «advenue », comme le souligne Platon, c'est qu'elle est le résultat d'une progression, d'une dynamique, d'un mouvement, qu'on voit bien dans les exemples qu'il donne et qui sont tous des exemples d'états transitoires : la santé, la beauté, pour être indéniables lorsqu'elles apparaissent, n'en sont pas moins des états transitoires. Elles manifestent un « équilibre impondérable », comme dirait Saint-Denys Garneau.

Je vais pousser un cran plus loin la réflexion, en me fondant cette fois sur Gadamer, qui commente ce passage du Philèbe de Platon. Gadamer y distingue « deux arts de mesurer» (1996, p. 197), qui correspondent au peras et à l'ousia nommés plus haut. Il rappelle que l'ousia est qualifiée par Platon d'« exactitude elle-même » et il précise ensuite :

Cela n'est pas en tout cas l'exactitude pure que l'on rencontre dans les nombres et les mesures et qui fait toute la dignité des mathématiques. Il ne faut pas voir dans cette expression [...] une allusion à la métaphysique des nombres chez Platon. Ce serait rater complètement la cible. Il y est d'ailleurs expressément précisé de quoi il s'agit lorsqu'il est question de l'« exactitude même», notamment de la juste mesure, du convenable, de l'instant propice et de ce qu'il faut faire. Cela se trouve justement appelé « le juste milieu entre deux contraires ». (Gadamer, 1996, p. 197)

Pour préciser ce que Platon cherche à définir alors, il se penche sur la question de l'être et sur le fait que l'être est cette chose qui « advient », ce qu'on a vu plus tôt dans l'expression d'ousia advenue. Il voit là toute la nouveauté de Platon par rapport aux pythagoriciens, qui distinguaient déjà l'illimité du limité, soit les deux premières catégories chez Platon. Il souligne que Platon utilise plusieurs expressions pour définir cette troisième catégorie, comme la «venue à l'être », l'« être devenu ». « De cette manière, [précise Gadamer,] il insiste encore davantage sur l'unité du devenir et de l'être. » (p. 202) Et c'est en précisant cette idée que Gadamer en vient à considérer l'œuvre d'art, dans une formule qui me semble essentielle : « Le pas qui mène du devenir à l'être laisse à l'être quelque chose de son être-devenu. [...] 
L'être ressort du devenir. [...] Nous reconnaissons ici l'expérience fondamentale que nous faisons face à l'œuvre d'art lorsque nous disons "il en est ainsi" - c'est “juste”. » (p. 202-203) Pour reprendre mon exemple plus tôt, je dirais que dans la santé, perçue comme un être, on voit encore les traces d'un « devenir » qui a mené à cet état, des traces d'une « mise en santé » ou d'une «mise en forme » qui a mené à cet état d'équilibre perçu positivement; et c'est précisément les traces de ce devenir qui nous font dire : «c'est cela, c'est visible, cet être est en santé ». Et c'est aussi ce qui advient dans l'œuvre d'art, et peut-être même là plus que partout ailleurs : on y perçoit les traces d'un travail, d'un travail qui est «advenu », certes, mais qui, grâce à lui, nous fait dire « c'est cela » ou, plus couramment, « c'est beau », ou « c'est juste, c'est ce que je voulais dire ». À peu près toutes les querelles esthétiques ont porté sur ce sujet, sur la somme de travail requise pour produire une œuvre, ou sur l'inconvenance à trop montrer un travail qui apparait alors inachevé. En posant l'être-devenu ou l'être-advenu, Platon montrait la nécessaire connivence qu'il y avait entre la production et le produit, entre l'infini et le fini. Ce devenir est si bien imbriqué à l'être qu'il tend à disparaître; et c'est bien pourquoi le travail artistique achevé se fait oublier lorsque l'œuvre l'a parfaitement intégré.

Je vais citer une dernière fois le commentaire de Gadamer, un passage assez long mais qui a l'avantage de nous rapprocher de nos préoccupations :

Tentons d'appliquer ceci à l'art. Nous ne nous demandons pas tant ce qui en ressort ici ou ce qui se montre. Nous disons simplement que «ça » ressort (" es » kommt heraus). Et nous le disons aussi bien dans le cas de l'image que dans celui du langage et de sa puissance poétique. Nous y faisons une expérience. Ce «faire» ne veut pas dire à proprement parler que nous faisons quelque chose, mais bien que quelque chose nous apparait lorsque nous comprenons correctement. Cela ne veut pas dire, mais pas du tout, que nous y introduisons ou que nous y lisons quelque chose qui ne s'y trouvait pas déjà. Par la lecture, nous en tirons plutôt quelque chose qui s'y trouve déjà et de façon telle que ça ressort. (p. 204)

Je rappellerai à ce titre une des Cent phrases pour éventails de Claudel, probablement la plus connue, qui dit : «Il faut qu'il y ait dans le poème un nombre tel qu'il empêche de compter » 
(p. 729). Le nombre est visible, il « ressort » comme dit Gadamer, mais il est « advenu » et s'est dégagé du décompte factuel pour saisir le lecteur dans son évidence. Ainsi apparait, à mon sens, l'autre mesure de la brièveté, sa juste mesure, toujours liée au sujet qui la perçoit sans pour autant tomber dans un aléatoire indéfini. La brièveté est cette chose qui « ressort » et qui « empêche de compter ».

Mais on le voit, j'ai dans cette discussion tranquillement débordé le cadre strict du discours et du langage; Gadamer s'intéresse à l'art en général, et j'ai plus tôt donné des exemples qui concernent la vie elle-même, la santé, la beauté ou les convenances sociales. Je vais à ce titre revenir au texte de Dessons, pour y noter une exclusion qui me semble on ne peut plus intrigante, et signe, là plus qu'ailleurs, d'un parti pris qui se dissimule mal. Dessons y fait référence par deux fois au texte fondateur de Sénèque, sur La brièveté de la vie, mais pour aussitôt l'exclure. La première mention, la plus étrange, se situe dans le relevé lexical que propose Dessons, lorsqu'il s'intéresse au terme «bref» : "À l'intérieur de la famille du mot "bref", la distribution des termes n'est d'ailleurs pas égale. Bref est d'une extension d'emploi relativement plus large - on parle, après Sénèque, d'une "vie brève" - que l'adverbe brièvement [...]. » (2015, p. 47) La remarque est étrange puisqu'on s'attendrait à voir Sénèque convoqué, non pour l'adjectif «bref », mais plutôt pour le nom «brièveté », attendu que ce dernier terme est donné dès le titre de son ouvrage, De la brièveté de la vie, De Brevitate vitæ en latin. D'ailleurs on verra dans mon deuxième exemple que Dessons y reviendra plus loin. Mais on devrait au moins noter ici que ce qui est dit du «bref » peut être dit aussi de la «brièveté », puisque Sénèque emploie les deux. Mais le plus étrange est de faire de cette acception une "extension», traitée d'ailleurs dans une parenthèse, comme si elle ne correspondait pas aux significations premières et principales du terme. Or il ne s'agit pas seulement d'opposer à cette conception la préséance historique de Sénèque, mais surtout le 
sens qu'elle implique. On voit mal comment la «vie», catégorie générale s'il en est, peut apparaître comme une extension de la dimension, du langage et du discours, qui eux donneraient les sens premiers. Du reste, l'exposé ici est retors, en ce qu'il semble suggérer que l'adjectif «brève» est utilisé comme un adjectif classifiant, ce qui amènerait à dire que certaines vies seulement sont brèves. Le titre de Sénèque, et l'exposé qui suit, laissent plutôt supposer que la brièveté est un qualifiant de la vie, et que toute vie, quelle qu'elle soit, apparait brève.

La deuxième mention est certes moins étrange mais elle frappe par son aspect extrêmement lapidaire, puisqu'elle convoque des notions obscures sans les développer. Dessons y fait mention d'un ouvrage de Fédor Rostopchine intitulé Mes mémoires en dix minutes. Il place ce projet dans la suite de l'ouvrage de Sénèque: «Notons que, même si l'ouvrage de Rostopchine répondait en fait à une provocation, on peut le considérer, malgré tout, comme un possible document prolongeant la réflexion de Sénèque dans De Brevitate vitæ, dans le sens d'une interrogation entre le trop-de-vie et le si-peu-d'écrire.» (Dessons, 2015, p. 86) Je soupçonne ici une coquille et qu'on devrait plutôt lire dans la dernière expression le verbe « décrire » plutôt qu'« écrire »; il n’en reste pas moins que l'expression est étrange et mériterait des développements. Or ceux-ci auraient entrainé Dessons à définir la relation qualifiante qui s'établit entre la brièveté et la vie, et à plus forte raison entre la vie et le récit qu'on peut en faire, qui sera toujours, nécessairement, en-deçà de ce que la vie propose, que ce récit dure six cents pages ou six lignes, ou comme le dit malicieusement Rostopchine, vingt ans ou dix minutes.

Je vais ici m'attarder quelques instants à l'exposé de Sénèque, et voir ce qu'il peut nous apprendre sur la brièveté. Sénèque y condamne notre conception de la vie comme étant trop courte, ce qui revient à sous-entendre la proposition que « la vie est courte », selon la logique 
des degrés déjà définie. Car s'il va de soi que "mourir à 20 ans » est trop court, rien n'empêche d'appliquer la même logique à quelqu'un qui meurt à 80 ans en pleine santé. C'est pourquoi à partir d'un critère accidentel (mourir trop vite) il devient essentiel (on meurt toujours trop vite). Pour Sénèque par contre, toute vie est suffisante à nos besoins, désirs et actions, à condition qu'on ne perde pas notre temps en activités futiles. Il nomme ceux qui tombent dans ce travers les « occupés », soit ceux qui ne sont jamais satisfaits de leur temps imparti et qui se placent constamment en situation de manque par différents vices que condamne le stoïcien, comme l'ivresse, la luxure, l'avarice, la colère, la vanité, etc. À ceux-ci il oppose les sages qui, eux, ont constamment à leur disposition ce qu'il nomme du « loisir », où ils peuvent notamment lire les anciens, ce qui leur permet d'ajouter à leur temps propre le temps historique des ancêtres. On retrouve ici les départages que nous avons vus plus tôt et qui tendent à distinguer deux types de brièveté de la vie : ce qu'on aurait pu croire être la vie brève, au sens d'une vie écourtée avant l'heure, d'une vie qui voit la mort advenir trop tôt, est en fait qualifiée de vie « courte »; alors que c'est la vie bien remplie qui est « brève », celle dont le temps est non seulement suffisant mais aussi en surplus, puisqu'il permet le «loisir » de la sagesse, et que cette sagesse elle-même ajoute à notre temps le temps historique. En ce sens, la vie brève est celle qui est marquée par une « juste mesure », qui correspond encore au code éthique du stoïcisme. Cette nuance est visible lorsque Sénèque dit : «Tout le reste de son étendue n'est pas de la vie, mais du temps. » (p. 103) On voit bien ici les deux mesures qui sont en jeu, la juste mesure et la mesure chiffrable, la durée.

Or il me semble nécessaire de marquer une nuance, car j'ai mis face à face la vie courte (condamnée par Sénèque) et la mort; et qu'on pourrait croire que les « occupés » seraient, par exemple, plus conscients de la mort qui approche, contrairement aux sages qui ne s'en soucieraient guère. Or c'est tout l'inverse que dit Sénèque : «Quelle en est la cause [du mal des 
hommes]? Vous vivez comme si vous deviez toujours vivre, jamais votre fragilité ne vous vient à l'esprit.»(p. 106) Ainsi les occupés, loin d'être conscients de leur mortalité, de leur « fragilité » constitutive, sont au contraire ceux qui font tout pour l'oublier, qui s'occupent à des activités qui les confortent dans une idée d'immortalité qu'ils entretiennent. De là vient un mépris pour le temps; et ici Sénèque représente les hommes comme étant très soucieux de conserver leurs avoirs mais très insouciants pour le temps qui leur est alloué, et qu'ils dépensent largement. C'est pourquoi la conscience de notre mortalité, en nous faisant vivre pleinement chaque moment, nous fait prendre conscience du temps qui passe et du profit que nous pouvons en tirer. Mais c'est dire du même coup que derrière cette éthique, que j'espère n'avoir pas rendue trop lourde, se décèle une conception plus large, dont on peut dégager notamment un «art», au sens d'un «faire», d'une habilité à produire, ce dont les œuvres pourront témoigner. Car à bien comprendre Sénèque, on voit bien que la brièveté n'est ni dans l'activité humaine ni dans le temps, mais plutôt dans l'adéquation qui nous fait placer l'une dans l'autre. C'est ainsi qu'il précise que non seulement la vie peut être vécue pleinement, mais aussi chaque journée; et au fond, pour l'essentiel, c'est à cette petite échelle de la journée que se situe le loisir lorsque, ayant mené à terme toutes ses activités, le sage peut se délecter du savoir des anciens.

Je résumerai en ces termes. Ce qui peut être qualifié de « court » est la durée; la vie, elle, est un cours, une somme d'actions qui s'enchainent et se motivent l'une l'autre. Placée devant la mort, la fragilité de l'être humain et sa finitude, la vie devient alors brève lorsqu'elle apparait suffisante, de juste mesure, et qu'elle laisse en surplus un loisir qui servira au développement de la sagesse, qui elle-même bonifiera le temps de l'humain, en superposant à sa vie les vies du passé. C'est pourquoi je crois que s'il fallait trouver une figure qui traduise mieux que toute autre la brièveté, ce ne serait pas la litote, ou l'ellipse ou toute autre figure de 
condensation et de concision, comme on l'a longtemps cru. Ce serait plutôt l'hyperbate, cette figure consistant à ajouter un membre à une phrase ou à une proposition qui paraissait terminée, ce qui permet de mettre en évidence le terme ajouté. Au loisir de Sénèque j'associe donc l'hyperbate, et il existe au moins un usage lexical qui me permet ce lien : c'est l'adverbe «bref », qu'on utilise à la fin d'une période pour en introduire le résumé. L'expression est dérivée de l'adjectif et a d'abord signifié (à la moitié du XIII siècle) «dans peu de temps, bientôt», puis a été utilisée (1403) dans la forme en brief, avec le sens de « en peu de mots », puis seule (1450), selon l'usage que nous connaissons, comme synonyme de « en résumé, enfin ». La progression de l'expression me semble en soi intéressante, puisqu'elle montre d'abord une conscience de la fin, lorsque l'adverbe a désigné un temps postposé, et donc la conscience du temps qui nous sépare de ce terme («bientôt»). Et de là se dégage le souci de bien utiliser ses mots, de pratiquer sur eux une restriction qui sera en même temps une clarification. En combinant ces deux aspects, on voit bien que lorsque, à la fin d'une période, nous disons «bref», nous avons alors pleine conscience des mots qui suivront «bientôt »; nous voyons d'abord le terme, la fin du discours, et comment nous allons y toucher en résumant ce qui précède. L'adverbe «bref » se tient donc en parfait équilibre, dans une juste mesure, entre ce qui précède et ce qui suit; ce qui montre aussi, par ailleurs, une conscience aiguë du temps. C'est pourquoi l'élément rejeté, placé devant le «bref », est forcément mis en évidence, le terme crée l'attente d'une reprise de l'idée mieux formulée, plus claire, et pour tout dire en un mot, qui respectera la « juste mesure ».

\section{Exemples : de l'hyperbate à la légèreté, en passant par la chair}


Je vais pour terminer considérer trois exemples qui tenteront de résumer les aspects que j'ai présentés. Je vais commencer par un emploi du «bref » dans son mode adverbial que je viens de traiter, et qui me conduira vers l'hyperbate - hyperbate qui, dans cet exemple, est au moins double, sinon triple. Il provient d'un recueil récent d'Yves Bonnefoy, intitulé L’heure présente :

En bref, la vie quotidienne de la lumière, quelque chose comme une intimité surprise mais qui d'emblée voulait bien, et rassurait. La lumière était mon amie, elle resterait avec moi tout ce jour encore. (p. 36)

Pour commencer mon commentaire, je vais proposer une hypothèse, au fond toute simple. À savoir que l'adverbe «En bref » n’a pas ici seulement qu'une fonction syntaxique de transition, mais qu'on peut aussi y trouver un sens plus profond, qui le relierait à ce qui suit. Je fais l'hypothèse, en somme, que la suite de l'adverbe questionne aussi la brièveté elle-même, posée en tête. L'hypothèse peut sembler oiseuse mais elle est au fond justifiée par un usage courant, qui fait par exemple qu'on a préféré cette expression à d'autres du même type, comme «en résumé, en somme, en d'autres mots ». Qu'on dise que ces expressions auraient paru bien peu poétiques ne règle rien, puisqu'il faudrait alors décider ce qui fait la poéticité du « En bref », ce qui nous ramène à nos préoccupations.

Or ce lien avec la brièveté frappe d'emblée, dans cet extrait, dans la mesure où il reprend nombre des observations faites plus tôt. Le thème central de l'extrait est bien sûr la lumière, et il est bien de rappeler que la lumière apparait sous un régime tout entier régi par la brièveté. Dans son opposition à l'ombre et aux ténèbres d'abord, qui montre que la lumière est une chose finie constamment confrontée à son envers, comme la vie face à la mort. Par ailleurs, la course de la lumière est bien sûr liée à la temporalité, et particulièrement à son régime courant et quotidien de révolutions des jours : la lumière, c'est ce qui donne l'heure. Ce sens est bien sûr investi pleinement dans l'extrait, qui nous parle précisément d'une «vie quotidienne » et se termine par le « jour ». Or cette lumière n'apparait pas seule; on la voit ici 
en constante interaction avec le sujet, qui se l'approprie jusque dans son « intimité » et qui lui donne même une intention éthique, avec cette expression si particulière: "qui d'emblée voulait bien ». Il en fait son « amie », elle rassure et elle reste.

Mais il y a encore quelque chose d'autre dans cette lumière qui saisit le sujet, et pour mieux le comprendre, je vais revenir à cette écriture de l'hyperbate dont je parlais plus tôt. On peut d'emblée considérer que tout l'extrait est une hyperbate, dans la mesure où il est introduit par un «En bref». Et de fait, l'expression qui suit immédiatement, strictement nominale, et d'une portée très générale, presque définitoire, apparait bien comme un ressaisissement fulgurant de la pensée : « la vie quotidienne de la lumière ». Il y a là, si on peut me permettre, une grande vérité qui est énoncée, vérité peut-être banale mais non moins vérifiable. Mais voilà, la phrase n'est pas terminée et se poursuit malgré tout. C'est ce que j'appellerais la deuxième hyperbate de l'extrait, qui commence avec «quelque chose » et va jusqu'à la fin. Par sa présence même, cette hyperbate révèle un certain pathos, comme si la perfection de la définition donnée plus tôt, plutôt que d'éclairer le sujet et le satisfaire, provoquait chez lui une sorte d'impatience, une impulsion à continuer; comme si la fermeture de la proposition nominale, au fond, n'avait rien dit encore d'essentiel. Et de fait, ce qui fera son entrée magistrale après l'expression « quelque chose », c'est le sujet lui-même, et surtout l'humanité de la lumière. Car la lumière - et c'est à Gadamer ici que je pense tout particulièrement - ne fait pas qu'être là, elle advient, elle «ressort»; ou comme le dit le poème, elle est une intimité mais « surprise », elle veut mais « d'emblée ». Et c'est là tout à coup un autre sens de la lumière qui surgit, et que ne saisissait pas encore la définition nominale : c'est que son action se poursuit toujours, que dès qu'on croit l'avoir saisie, elle revient encore. Et c'est cela, précisément, ce que cette deuxième hyperbate exprime et signifie tout ensemble. 
Il reste à parler de la troisième hyperbate; c'est la plus simple stylistiquement, en ce qu'elle reprend son procédé le plus courant, soit l'ajout d'une conjonction de coordination à une proposition qu'on croyait complète. On la trouve dans le segment « et rassurait». Mais cette simplicité ne doit pas faire écran à ce qu'elle implique, qui me semble primordial. Le mot « bien » la précède et ce n'est pas innocent : «vouloir bien », c'est bien sûr « vouloir du bien, vouloir le bien de quelqu'un », mais c'est aussi « bien vouloir », c'est-à-dire vouloir selon une modalité efficiente, qui fait par exemple qu'il peut y avoir adéquation entre le vouloir et le pouvoir. Or qu'est-ce qui rassure dans la lumière? Le fait qu'elle soit présente chaque jour, comme un métronome régulier? Ou le fait qu'elle déborde continûment, le fait qu'elle donne plus que ce qu'on peut recevoir, le fait qu'on doive user de l'hyperbate pour au moins évoquer sa force excessive? Je penche bien sûr pour la deuxième option, et je remarque par ailleurs que cette dernière hyperbate introduit un verbe, conjugué à l'imparfait, qui est le temps long des actions continues; de ces actions qui, peut-être se poursuivent après qu'on en ait parlé, mais qu'on doit considérer comme du passé parce qu'on a décidé d'en parler. Or tous les verbes de l'extrait sont à l'imparfait, sauf le dernier, au conditionnel, dans une forme un peu particulière en français, puisqu'il désigne une action au passé; mais un passé qui aura été vécu dans l'incertitude, comme si le poète s'était dit constamment « cette lumière, va-t-elle rester avec moi tout le jour? » Oui, elle resterait, oui, elle devait rester. Mais « rester » non pas de façon statique, mais au contraire comme quelque chose qui pouvait s'arrêter, mais a décidé de rester « encore ». À cet excès, à ce surplus continu, Bonnefoy a ici donné les mots de l'amour, si présents dans l'«intimité », dans le fait de rassurer ou de se sentir ami. Car comme le dit Ricoeur (2008), l'amour n'est pas le domaine de la justice, mais celui d’un excès qui développe sa propre « juste mesure », mesure non quantifiable qu'aucun nombre ne peut saisir. 
Mon deuxième exemple est tiré de Saint-John Perse, un auteur dont on peut dire sans se tromper que les modalités du grand et de l'ample sont pour lui comme une seconde peau.

Les vents sont forts! la chair est brève!... Aux crêtes lisérées d'ors et de feux dans les lancinations du soir, aux crêtes ciliées d'aiguilles lumineuses, parmi d'étranges radiolaires,

N'est-ce toi-même tressaillant dans de plus pures espèces, avec cela d'immense et de puéril qui nous ouvre sa chance?... Je veille. J'aviserai. Et il y a là encore matière à suspicion... Qu'on m'enseigne le ton d'une modulation nouvelle! (p. 213)

Devant cet extrait, je me poserai encore une question toute simple : qu'est-ce qui en fait la grandeur et l'ampleur? Est-ce sa longueur, de celle qu'on attribue au verset, fournie par ces quarante-huit et ces soixante-huit syllabes? Ou n'est-ce pas plutôt l'accumulation des exclamations et des questions, un vocabulaire majestueux et recherché, et surtout la gradation rythmique et thématique des propositions, qui s'enflent et progressent jusqu'à une acmé finale? Je penche plutôt, encore là, pour la deuxième hypothèse. Même s'il n'y a pas à proprement parler de rejet sur le deuxième verset, on est quand même frappé que Saint-John Perse a tenu à marquer un suspens à la fin du premier, avant d'introduire une question, visible dès l'amorce du second verset, par l'inversion propre à la proposition interrogative, « N'estce ». Or c'est précisément ce que développe cet extrait : non une question en tant que telle, mais une posture questionneuse, faite d'inquiétude et d'attention soucieuse, un souci de l'avenir qui dépasse les certitudes, comme celles que peut nous donner la science, ici convoquée massivement par tout ce vocabulaire naturaliste et géologique, ces «crêtes lisérées » et « ciliées », ces « aiguilles », ces « radiolaires ».

Or cette posture questionneuse, visible par la syntaxe d'une proposition interrogative qui enjambe les deux versets, se répercute en plusieurs endroits, et dès l'amorce du premier verset. On y trouve deux exclamations parfaitement symétriques, de quatre syllabes chacune, qui, juxtaposées comme elles le sont, posent à l'être humain une question fondamentale. Que puis-je faire en ce monde, si «les vents sont forts » et que «ma chair est brève »? Comment 
puis-je confronter des forces qui dépassent tous mes moyens de saisie, toute cette science, toutes les meilleures volontés, toute mon énergie? Car la brièveté de la chair doit ici nous alerter. Il serait vain d'y chercher un hédonisme que condamnait Sénèque. On s'en convaincrait en remarquant qu'on trouve quelques lignes plus haut une mention des « os » de l'homme, soit bien sûr son squelette, et partant sa finitude, sa mortalité. Or il y a plus encore, car qu'est-ce, au fond, qu'une « chair brève »? Est-ce une chair qui dure peu de temps, nous plaçant alors dans le compte mathématique du corps qui vieillit et se dégrade? Ce serait oublier la présence massive de la temporalité du présent dans cet extrait, où presque tous les termes sont non seulement au présent, mais dans ce présent d'éternité qui participe lui aussi à l'ampleur de l'écriture persienne - et oublier encore ce verbe au futur, "J'aviserai », et l'expression finale, qui exprime un souhait. Ce n'est pas non plus, convoquant alors le sens spatial de la brièveté, une chair ou un corps de petite dimension, comme on peut dire d'un mouvement, d'une enjambée ou même d'un nez, qu'ils sont brefs. La proximité avec le mot « crêtes », qui répond à « brève » par une paronomase symétrique, et qui désigne pour sa part une «excroissance» tant zoologique que géologique, semble définitivement exclure cette possibilité. Mais une autre voie se dégage de cette confrontation, car l'excroissance de la «crête » peut être un désavantage ou même une malformation, pointant vers la monstruosité des êtres, ce que semble confirmer les tourments qu'évoquent les « lancinations » qui suivent. Or j'aimerais ici reprendre deux remarques faites plus haut, à savoir que la brièveté serait un «être advenu », la trace d'un devenir toujours visible dans l'être. Et à savoir que la brièveté m’apparait comme un qualifiant de la vie, plutôt qu'un classifiant. Ce sont ces deux phénomènes qui sont visibles ici, à mon sens, et qui font de la brièveté non pas un attribut accidentel de la chair, mais au contraire une qualité qui la définit dans son essence propre. Brièveté qui en donne la texture et la marque, qui se présente comme la finitude en marche, 
entrainée et enchainée, dirais-je, vers le futur, inexorablement, mais qui de ce fait rend la chair elle-même pleinement visible et lui donne sa "véhémence ontologique », comme dirait encore Ricoeur (1975). Et c'est pourquoi l'opposition qu'on a perçue entre les «vents forts » et la « chair brève », pour nous sembler d'un possible pathétique, n'a rien d'accidentel; et pourquoi, plutôt que d'opposition, nous devrions plutôt parler de complémentarité, d'une nécessité duelle entre ces deux versants, qui fait toute l’ampleur déployée de cet extrait.

Et on notera alors la marque qu'imprime à cet extrait sa structure duelle. L'image des « crêtes » est répétée deux fois, on l'a déjà dit, tout comme on a parlé de l'enjambement de la proposition au centre de l'extrait. Mais deux autres couples vont nous intéresser. Le premier reprend en raccourci la symbiose conflictuelle qu'on percevait entre les vents et la chair : il qualifie l'interlocuteur ou l'interlocutrice, lorsqu'il lui est dit que l'accompagne «cela d'immense et de puéril qui nous ouvre sa chance ». L'immense rappelle bien sûr la force des vents alors que le puéril réfère à la brièveté de la chair. Or c'est là, remarquons bien, une « chance », et non plus une fatalité. Car cela permet deux postures, deux actions, et c'est alors le second couple que je convoque : «Je veille. J'aviserai. » Deux propositions lapidaires, que tout distingue dans cet extrait : le sujet personnel, la différence des temps de verbe, et bien sûr leur brièveté, au sens à la fois de ce qui est court et de ce qui est bref. Ces verbes participent pleinement de la posture questionneuse que je décrivais plus tôt : ils montrent un sujet en pleine tension, qui veille non pas un mort, mais qui veille parce qu'il est mortel, et le sera toujours, pour ainsi dire, il est aux aguets et se tient prêt à témoigner de ce qu'il verra. Or ce témoignage me permettra de parler d'une autre signification de la brièveté dont je n'ai pas parlé. En effet, en considérant l'expression « la chair est brève », je ne me suis pas demandé s'il s'agissait d'une brièveté discursive, langagière ou poétique. Ce sens aurait paru au moins étonnant. Mais pourtant, l'extrait se termine par une invocation à peine voilée à marcher dans 
les voies du poème, c'est-à-dire à trouver «le ton d'une modulation nouvelle ». Face à la brièveté de la chair, face à la confrontation entre chair et vents, quelle est cette « chance » que trouve le poète? Celle de trouver une parole qui puisse se moduler à cette expérience. Mais bien davantage encore : celle de la parole elle-même, du langage et de l'écriture, que nous avons forgés précisément pour répondre à notre brièveté, et pour donner une mesure à ce monde qui nous dépasse.

Ce qui est grand, majestueux, sublime dans la voix de Saint-John Perse, c'est avant tout la brièveté de la chair d'un être humain.

Je vais terminer ce court survol tout en douceur, par un extrait de François Cheng :

Il fait bon sur une margelle

Aux tièdes mousses s'attarder

Uni à la fraîcheur de l'ombre

Contempler le dernier rayon

Du couchant qui tisse en images

- avec les aiguilles des pins

Sa brève légende dorée (p. 58)

Je vais dire peu de choses de ce court extrait et j'assume d'emblée l'impressionnisme de ces quelques remarques naïves et sans prétention. Je veux seulement remarquer que la sensation immédiate et générale que me donne cet extrait en est une de légèreté. Je lis, je vois bien que cette « légende dorée » est qualifiée de « brève », mais sans contrôle de ma part, j'entends et je comprends qu'elle est « légère ». Bien sûr tout le reste de l'extrait concourt à préparer cette légèreté, et je peux même dire qu'elle s'impose de plus en plus, jusqu'à l'image du calligraphe ou du dessinateur japonais qui trace, «avec les aiguilles des pins », en touchant à peine la surface me semble-t-il, sa légende merveilleuse.

Est-ce, donc, que la brièveté peut-être associée à la légèreté? - et ce sera là ma dernière question naïve. À priori, il n’y a pas loin entre une «brève légende dorée » et les « Mémoires en dix minutes » que j'évoquais plus tôt. Et pourtant on ne peut s'arrêter là, car il 
faut ici considérer le passage entre l'écrit et le visuel, puisque cette légende brève n'est pas écrite, mais tissée - et d'ailleurs plus tissée que dessinée ou peinte, et on peut déjà voir dans cette nuance comme un résumé, une esquisse à grands traits qui ne s'attarde qu'aux lignes saillantes. Et encore, cette « texture » de la légende n'est elle-même que métaphorique, car il s'agit ici de décrire les arborescences du paysage, saisies en un moment particulièrement sublime. Or ce qui frappe à ce niveau, c'est comment cette scène s'inscrit dans un temps extrêmement long, presque statique : chaque chose s'étire, le sujet est posté dans une attente sans faille, tout l'insensible de la scène ne lui échappe pas, jusqu'à ce que se profile un peu d'or, comme les tisons vifs d'un feu qui s'éteint.

Est-ce que la brièveté est légère? Oui si, comme le dit Gadamer, la brièveté est ce qui ressort, ce qui se détache d'un fond indifférent, se tend légèrement vers soi et attend qu'on l'observe. Cette brièveté ne se tient pas sur un plan vertical, entre la terre et le ciel; ce n'est pas cette légèreté qui est visée ici. Elle se tient sur un plan horizontal, car c'est sur ce plan qu'apparait l'or des rayons du soleil couchant; elle se tient là quelques secondes, quelques minutes, entre moi et l'horizon. Et face à moi, qui l'attendait.

\section{Bibliographie}

Bonnefoy, Yves (2014), L’heure présente et autres textes, Paris, Gallimard, coll. « Poésie ».

Cheng, François (2005), À l'orient de tout. OEuvres poétiques, Paris, Gallimard, coll. « Poésie ».

Claudel, Paul (1957), CEuvre poétique, une édition de Jacques Petit, Paris, Gallimard, coll. « Bibliothèque de la Pléiade».

DelcomminetTE, Sylvain (2005), « La juste mesure. Étude sur les rapports entre le politique et le Philèbe », Les Études philosophiques, no 74 (3), p. 347-366, https://www.cairn.info/revue-les-etudes-philosophiques-2005-3-page-347.html.

DESSONS, Gérard (2015), La voix juste. Essai sur le bref, Paris, Manucius, coll. « Le marteau sans maître ». 
Formes brèves. De la gnômè à la pointe : métamorphose de la sententia, La Licorne, $\mathrm{n}^{\circ} 3$, février 1979.

FOYARD, Jean (2001), La forme brève, Dijon, Presses universitaires de Dijon, coll. « Le texte et l'édition ».

GADAMER, Hans-Georg (1996), La philosophie herméneutique, traduction de Jean Grondin, Paris, Presses universitaires de France, coll. «Épiméthée ».

LEPLATRE, Olivier (éd.) (2005), La forme brève, Paris, Gallimard, coll. « La Bibliothèque Gallimard ».

MessinA, Simone (dir.) (1996), La forme brève, Paris, Honoré Champion, coll. "Textes et études ».

Montandon, Alain (1992), Les formes brèves, Paris, Hachette Supérieur, coll. «Contours littéraires ».

Ricoeur, Paul (1975), La métaphore vive, Paris, Seuil, coll. « L’ordre philosophique ».

Ricoeur, Paul (2008), Amour et justice, Paris, Seuil, coll. « Essais ».

RouкномоvsкY, Bernard (2005), Lire les formes brèves, Paris, Armand Colin, coll. « Lettres sup. ».

Saint-John Perse (1982), CEuvres complètes, Paris, Gallimard, coll. «Bibliothèque de la Pléiade ».

SEneque (2005), La vie heureuse. La brièveté de la vie, une édition de Pierre Pellegrin, une traduction de José Kany-Turpin et Pierre Pellegrin, Paris, Flammarion, coll. « GF ».

\title{
Résumé
}

Cet article entend lier la question de la brièveté à l'écriture poétique, non du point de vue des formes brèves ou du genre, mais d'un point de vue plus philosophique, lié à la finitude. Dans un premier temps nous discutons les thèses de l'essai de Dessons (2015), pour tenter d'y apporter un complément, avec la notion de l'ousia, de «l'être-advenu » notamment, que nous empruntons au Philèbe de Platon. Par la suite, et suivant Sénèque, nous proposons de faire de l'hyperbate la figure de la brièveté. Dans une seconde partie, nous tentons d'appliquer ces notions à l'étude de trois extraits poétiques, de Bonnefoy, Saint-John Perse et Cheng, chez qui nous trouvons, dans l'ordre : une écriture de l'hyperbate, de la finitude et de la légèreté.

\begin{abstract}
This paper aims to connect the poetic writings to the brevity, not as a short form or as a genre, but as the philosophical quest of our finitude. In first, we discuss the thesis of Dessons (2015)
\end{abstract}


and try to complete them with the Plato's Philebus, especially the notion of ousia, the " becoming and being ». Afterward, with a discussion of Seneque, we propose to consider the hyperbaton as the brevity's figure. In a second part, we transpose these notions to the analysis of three poetics extracts, from Bonnefoy, Saint-John Perse and Chang. We expose, in order, their hyperbate's, finitude's and lightnesses scriptures. 